\title{
Low Diameter Algebraic Graphs
}

\author{
Bui Xuan Hai ${ }^{1,2}$, Bui Xuan Binh Minh ${ }^{3}$, Le Van Chua ${ }^{1,2,4}$, and \\ Mai Hoang Bien ${ }^{1,2}$ \\ 1 University of Science, Ho Chi Minh City, Viet Nam \\ 2 Vietnam National University, Ho Chi Minh City, Viet Nam \\ [bxhai,mhbien] @hcmus.edu.vn; lvchua.tag@moet.edu.vn \\ ${ }^{3}$ LIP6 (CNRS-SU UPMC), Paris, France \\ buixuan@lip6.fr \\ 4 An Giang University, An Giang, Viet Nam
}

\begin{abstract}
Let $D$ be a division ring, $n$ a positive integer, and $\mathrm{GL}_{n}(D)$ the set of invertible square matrices of size $n$ and values in $D$, called the general linear group. We address the intersection graph of subgroups of $\mathrm{GL}_{n}(D)$ and prove that it has diameter at most 3. Two particular cases of its induced subgraphs are then investigated: by cyclic subgroups, and by almost subnormal subgroups. We prove that the latter case results in a connected graph whose diameter is sharply bounded by 2 . In the former case, we completely characterise the connectivity of the induced graph with respect to $D$, where, in case of connectivity, we prove that it has diameter at most 7 in general, and at most 5 if $D$ is a locally finite field of characteristic not 2 different from $\mathbb{F}_{3}$ and $\mathbb{F}_{9}$.
\end{abstract}

Keywords: division ring, intersection graph, graph connectivity

\section{Introduction}

Geographically, human activities can naturally be embedded into an Euclidean space $\mathbb{R}^{n}$ with $n=3$ or $n=2$, by extending infinitely the region on Earth under study and by projecting latitude, longitude and altitude coordinates of that region on the space. We formalise graphs arising from Euclidean spaces as in the sense of intersection graphs [18]: given a set of geometric objects in the space, the corresponding intersection graph has these objects as vertices, and the vertices are adjacent if and only if the objects have a non-trivial nonempty intersection. When the space is a plane, that is, $n=2$, intersection graph theory sows the way for important research results: social network simulations can stem from the assumption where adjacency likeliness increases in function of the geographic distance of disks in the space 21, planar graph characterisation and coloring can rely on intersection of segments in the space [620], and RNA structures can benefit from approximations using the proximity of intervals (and pairs of intervals) on a line [712.

\footnotetext{
* Supported by Courtanet - Sorbonne Université convention C19.0665 and ANRT grant 2019.0485.
} 
More generally, geometric graphs are well captured in the $(d, e)$-subspace intersection model proposed in Laison-Qing's classification [17. Following these termes, $d$ denotes the space dimension and $e$ the dimension of the geometric objects: Penrose's random geometric networks belong to $(2,2)$-subspace intersection graphs, Scheinerman planar graph characterisation to $(2,1)$-subspace intersection graphs, and RNA approximations to $(1,1)$-subspace intersection graphs.

Generalising further, an important step has been taken by Yaraneri's studies on submodules [22]. Here, the real field $\mathbb{R}$ is left behind in favor of ring structures. Rings are important because cryptosystems can rely on hardness results of their isomorphisms [16] in order to define new cybersecurity schemes [413. Historically, studying intersection graphs of elements of a ring has been initiated by Bosák [3] and further developed with Csákány-Pollák's theorems [8]. Following these works, many research efforts have been put in studying the structure of intersection graphs stemming from group structures [5]11. However, to the best of our knowledge, very little results are known for the most general case of a division ring $D$. This is precisely the shortage where Yaraneri's results help rectifying when studying the intersection graph of submodules of a (left/right) module over a division ring $D$. For a simpler introduction, let us ignore the case of infinite dimension, and define $D_{R}^{n}$ as a right $D$-module of dimension $n$. Then, in a nut shell, column vectors of module $D_{R}^{n}$ over ring $D$ are natural extensions of vectors of space $\mathbb{R}^{n}$ over the field $\mathbb{R}$ of real numbers. This way, one can retrieve most of the nice topology results from the segments of a plane in the submodules of a module 22. However, Yaraneri left unstudied the extension of column vectors to matrices over $D$. In particular, the graph structure of ring morphisms is not well understood. In this paper, we help paving the road toward this research direction with invertible square matrices.

More precisely, we lift the intersection model of geometric graphs to the algebraic graph defined by non-trivial intersections of proper subgroups of the general linear group $\mathrm{GL}_{n}(D)$, that we denote by graph $\Gamma\left(\mathrm{GL}_{n}(D)\right)$. Here, the general linear group is the set of invertible square matrices of size $n$. Its subgroups are sets of invertible square matrices closed under multiplication and inversion. When $n \geq 2$ and $D=F$ is a field with at least 3 elements, Bien and Viet recently proved that $\Gamma\left(\mathrm{GL}_{n}(F)\right)$ has diameter either 2 or $3[2]$.

In Section 2, we extend Bien-Viet's theorem from fields to arbitrary division rings, and prove furthermore for $n=1$ that $\Gamma\left(\mathrm{GL}_{1}(D)\right)$ with $D$ infinite has diameter at most 3 , and exactly 2 when $D$ is so-called weakly locally finite. Proving these results requires a careful analysis of namely the non-central subgroups of $\mathrm{GL}_{n}(D)$. Thus, we push further in Section 3 our analysis on prominent ring structures with a non-trivial center. One of our major results is that they need not be complex: the non-central almost subnormal subgroups, when exist, always induce a clique, while the almost subnormal subgroups induce a subgraph of diameter bounded by a surprisingly sharp 2 . We then investigate the subgraph $\Gamma_{c}\left(\mathrm{GL}_{n}(D)\right)$ induced by cyclic subgroups of $\mathrm{GL}_{n}(D)$, and found a very close relationship with the proper power graph $P^{*}\left(\mathrm{GL}_{n}(D)\right)$. This helps characterising the graph's connectivity, in which case we show that it has diameter at most 7 . 
Examining particular cases, when $n=2$ and $D$ is either Galois fields $\mathbb{F}_{3}$ or $\mathbb{F}_{9}$, we prove that $\Gamma_{c}\left(\mathrm{GL}_{n}(D)\right)$ is not connected. Surprisingly, when $n \geq 3$ and $D$ is either above Galois fields, we result in that $\Gamma_{c}\left(\mathrm{GL}_{n}(D)\right)$ is connected, therefore, has diameter at most 7 . Except for the previous cases, when $n \geq 2$ and $D=F$ is a locally finite field of characteristic not 2, we prove that the diameter of $\Gamma_{c}\left(\mathrm{GL}_{n}(D)\right)$ is at most 5. In Section 4, we close the paper with concluding remarks and perspectives for research on algebraic graphs. By space restriction, properties marked with $(\star)$ are given without a proof.

\section{General Linear Groups}

For a loopless simple undirected (not necessarily finite) graph $\Gamma$, we note $V(\Gamma)$ its vertex set, $E(\Gamma) \subseteq\left(\begin{array}{c}V(\Gamma) \\ 2\end{array}\right)$ its edge set, and $u \sim v$ every edge $\{u, v\} \in E(\Gamma)$. A path joining $u$ to $v$ of length $k$ is a sequence of $k+1$ distinct vertices $u=v_{0} \sim$ $v_{1} \sim v_{2} \sim \cdots \sim v_{k}=v$. The geodesic distance $\mathrm{d}(u, v)$ between $u$ and $v$ is $+\infty$ if no such path exists, and the minimum length of such a path otherwise. Graph $\Gamma$ is connected if there exists a path joining any pair of its vertices. When $\Gamma$ is connected, if moreover the set $\{\mathrm{d}(u, v) \mid u, v \in V, u \neq v\}$ is bounded, then we define the diameter of $\Gamma$ as $\operatorname{diam}(\Gamma)=\max \{\mathrm{d}(u, v) \mid u, v \in V, u \neq v\}$.

The intersection graph $\Gamma(G)$ of a group $G$ has as vertex set the non-trivial proper subgroups of $G$. Two vertices of $\Gamma(G)$ are adjacent if and only if their intersection is not reduced to $\left\{1_{G}\right\}$. A division ring $D$ is a ring where every non-zero element is invertible. In particular, $D$ can as well be non-commutative or commutative, where it is called a field. The general linear group $\mathrm{GL}_{n}(D)$ is the set of invertible square matrices of size $n \geq 1$ and values in $D$. The center of $D$ is its subset containing every element $c \in D$ with $c x=x c$ for every $x \in D$. When $n=1$, the general linear group coincides with the multiplicative group, and is noted $D^{*}=\mathrm{GL}_{1}(D)$. The Galois field with $q$ elements is noted $\mathbb{F}_{q}$.

Theorem $1(\star)$. Let $D$ be a division ring whose center $F$ contains at least 3 elements, and $n$ an integer. When $n=1$, assume additionally that $D$ is infinite. Then, for every $n \geq 1$, the intersection graph $\Gamma\left(\mathrm{GL}_{n}(D)\right)$ of subgroups of the general linear group $\mathrm{GL}_{n}(D)$ has diameter at most 3 . Moreover, if $n \geq 2$, then $\Gamma\left(\mathrm{GL}_{n}(D)\right)$ has diameter at least 2 . When $n=1$, the requirement for $D$ to be infinite cannot be relieved: $\Gamma\left(\mathbb{F}_{7}^{*}=\mathrm{GL}_{1}\left(\mathbb{F}_{7}\right)\right)$ is not a connected graph.

In the sequel, we compare Theorem 1 with known results when $D=F$ is a field [2]. A division ring $D$ with center $F$ is weakly locally finite if for every finite subset $S$ of $D$ the division subring $F(S)$ generated by $S$ over $F$ is a finite dimensional vector space over its center [9]. Theorem 2 below generalizes [2, Theorem 4.2], which holds for $n \geq 2$ and $D=F$ a field. Both theorems contrast sharply with the finite case of a field $D=F$ with $D^{*}=\mathrm{GL}_{1}(D)$ of prime order, where the diameter is exactly 3 instead of 2 [2, Proposition 3.5]. We stress that every locally finite division ring is weakly locally finite [14, Theorem 4], however, the converse is not necessarily true, for infinitely many instances [10].

Theorem $2(\star)$. Let $D$ be an infinite weakly locally finite division ring. For every $n \geq 1, \Gamma\left(\mathrm{GL}_{n}(D)\right)$ has diameter exactly 2 . 


\section{Almost Subnormal Subgroups and Cyclic Subgroups}

A subgroup $N$ of a group $G$ is almost subnormal if there exists a sequence of subgroups $N=N_{r}<N_{r-1}<N_{r-2}<\cdots<N_{1}<N_{0}=G$ such that for every $r \geq i>0$, either $N_{i}$ is a normal subgroup of $N_{i-1}$, noted $N_{i} \triangleleft N_{i-1}$, or the index $\left[N_{i-1}: N_{i}\right]$ is finite, as in the sense of [15]. Following a previous work [19], we conjecture that the almost subnormal subgroups of $\mathrm{GL}_{n}(D)$ induce a clique in $\Gamma\left(\mathrm{GL}_{n}(D)\right)$. When $n \geq 2$, 19, Theorem 3.3] implies that the almost subnormal subgroups of $\mathrm{GL}_{n}(D)$ are also normal subgroups, and the conjecture follows. Unfortunately, for $n=1$ the almost subnormal subgroups are not necessarily normal subgroups, leaving the conjecture whether they form a clique in $D^{*}=$ $\mathrm{GL}_{1}(D)$ unanswered. Our main contribution in this topic is a tailored proof for below Theorem 3 bridging the gap for $n=1$ with a positive answer.

Theorem $3(\star)$. For any division ring $D$, the family of non-central almost subnormal subgroups of $D^{*}=\mathrm{GL}_{1}(D)$ is closed under intersection. It induces a clique in $\Gamma\left(D^{*}\right)$ when not empty. The family of almost subnormal subgroups of $D^{*}$ induces a connected subgraph of diameter at most 2 . The bound is sharp.

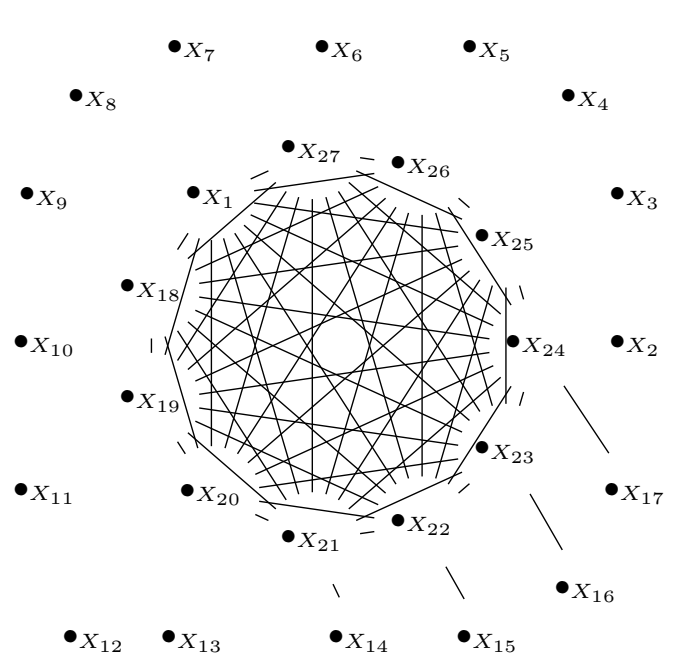

Fig. 1. Graph $\Gamma_{c}\left(\mathrm{GL}_{2}\left(\mathbb{F}_{3}\right)\right)$.

The cyclic subgroup intersection graph $\Gamma_{c}(G)$ of a group $G$ is the subgraph of $\Gamma(G)$ induced by all cyclic subgroups of $G$. A field is said to be locally finite if every finitely generated subfield over its prime subfield is finite. Theorem 4 below goes over our results on cyclic subgroups. In particular, for every $n \geq 3$ we found that $\Gamma_{c}\left(\mathrm{GL}_{n}\left(\mathbb{F}_{3}\right)\right)$ has diameter at most 7 . However, we result surprisingly in that $\Gamma_{c}\left(\mathrm{GL}_{2}\left(\mathbb{F}_{3}\right)\right)$ is disconnected, $c f$. Figure 1 . 
Theorem $4(\star)$. Let $n \geq 2$ be an integer and $D$ a division ring of characteristic not 2. If $\Gamma_{c}\left(\mathrm{GL}_{n}(D)\right)$ is connected, then $D$ is a locally finite field. Conversely, if $F$ is a locally finite field whose characteristic is not 2, then the following statements hold.

1. Both $\Gamma_{c}\left(\mathrm{GL}_{2}\left(\mathbb{F}_{3}\right)\right)$ and $\Gamma_{c}\left(\mathrm{GL}_{2}\left(\mathbb{F}_{9}\right)\right)$ are disconnected.

2. If $n \geq 3$, then both $\Gamma_{c}\left(\mathrm{GL}_{n}\left(\mathbb{F}_{3}\right)\right)$ and $\Gamma_{c}\left(\mathrm{GL}_{n}\left(\mathbb{F}_{9}\right)\right)$ have diameter at most 7 .

3. In all other cases, $\Gamma_{c}\left(\mathrm{GL}_{n}(F)\right)$ has diameter at most 5 .

The characteristic requirement cannot be omitted: $\Gamma_{c}\left(\mathrm{GL}_{2}\left(\mathbb{F}_{2}\right)\right)$ is disconnected.

Theorem 4 also helps answering positively to below Question 1 when the group is the general linear group. The power graph $\mathrm{P}(G)$ of a group $G$ has $G$ as vertex set, and two distinct vertices are adjacent if and only if one of them is a positive power of the other. The proper power graph $\mathrm{P}^{*}(G)$ of $G$ is the subgraph of $\mathrm{P}(G)$ induced by all non-identity elements of $G$. Note for a periodic group $G$ that $\mathrm{P}(G)$ has diameter at most 2 since every vertex is connected to $1_{G}$. However, $\mathrm{P}^{*}(G)$ is not necessarily connected, for instance when $G=S_{3}$ is the symmetric group of degree 3 .

Question 1. [1, Question 39] Which groups do have the property that the proper power graph is connected?

Theorem $5(\star)$. Given an integer $n \geq 2$ and let $D$ be a division ring of characteristic not 2 . Then, the following conditions are equivalent:

1. The graph $\mathrm{P}^{*}\left(\mathrm{GL}_{n}(D)\right)$ is connected.

2. The graph $\Gamma_{c}\left(\mathrm{GL}_{n}(D)\right)$ is connected.

3. $D=F$ is a locally finite field, and $\mathrm{GL}_{n}(F) \neq \mathrm{GL}_{2}\left(\mathbb{F}_{3}\right) ; \mathrm{GL}_{2}\left(\mathbb{F}_{9}\right)$.

\section{Conclusion and perspectives}

We show that most intersection graphs of subgroups of the general linear group over a division ring have a low diameter. We hope this can help understanding ring morphisms and leave open the question whether cyclic subgroups induce clique-like components, like those of $\mathrm{GL}_{2}\left(\mathbb{F}_{3}\right)$ presented in Figure 1.

Acknowledgements: We are grateful to the anonymous reviewers for their helpful insights and pointers.

\section{References}

1. Aalipour, G., Akbari, S., Cameron, P.J., Nikandish, R., Shaveisi, F.: On the structure of the power graph and enhanced power graph of a group. Electronic Journal of Combinatorics, 23, 2017. \#P3.16. doi:10.37236/6497

2. Bien, M.H., Viet, D.G: Intersection graphs of general linear groups. Journal of Algebra and Its Applications. In press. doi:10.1142/S0219498821500390 
3. Bosák, J.: The graphs of semigroups. In: Theory of Graphs and Its Applications, Czech. Acad. Sci., pages 119-125, 1964.

4. Calderini, M., Civino, R., Sala, M.: On properties of translation groups in the affine general linear group with applications to cryptography. Journal of Algebra, 569:658-680, 2021. doi:10.1016/j.jalgebra.2020.10.034

5. Chakrabarty, I., Ghosh, S., Mukherjee, T.K., Sen, M.K.: Intersection graphs of ideals of rings. Discrete Mathematics, 309:5381-5392, 2009. doi:10.1016/j.disc.2008.11.034

6. Chalopin, J., Gonçalves, G.: Every planar graph is the intersection graph of segments in the plane. In: 41th Symposium on Theory of Computing Conference, ACM, pages 631-638, 2009. doi:10.1145/1536414.1536500

7. Crochemore, M., Hermelin, D., Landau, G.M., Rawitz, D., Vialette, S.: Approximating the 2-interval pattern problem. Theoretical Computer Science, 395(2-3):283-297, 2008. doi:10.1016/j.tcs.2008.01.007

8. Csákány, B., Pollák, G.: The graph of subgroups of a finite group. Czechoslovak Mathematical Journal, 19:241-247, 1969.

9. Deo, T.T., Bien, M.H., Hai, B.X.: On radicality of maximal subgroups in $\operatorname{GL}(n, D)$. Journal of Algebra, 365:42-49, 2012. doi:10.1016/j.jalgebra.2012.04.027

10. Deo, T.T., Bien, M.H., Hai, B.X.: On weakly locally finite division rings. Acta Mathematica Vietnamica, 44:553-569, 2019. doi:10.1007/s40306-018-0292-x

11. Freedman, S.D.: The intersection graph of a finite simple group has diameter at most 5. Archiv der Mathematik, 2021. doi:10.1007/s00013-021-01583-3

12. Golumbic, M.C.: Interval graphs and related topics. Discrete Mathematics, 55:113121, 1985. doi:10.1016/0012-365X(85)90039-1

13. Grochow, J.A., Qiao, Y.: On the complexity of isomorphism problems for tensors, groups, and polynomials I: Tensor isomorphism-completeness. In: 12th Innovations in Theoretical Computer Science Conference, volume 185 of LIPIcs, pages 31:1-31:19, 2021. doi:10.4230/LIPIcs.ITCS.2021.31

14. Hai, B.X., Ngoc, K.N.: A note on the existence of noncyclic free subgroups. Archiv der Mathematik, 101:437-443, 2013. doi:10.1007/s00013-013-0576-2

15. Hartley, B.: Free groups in normal subgroups of unit groups and arithmetic groups. Contemporary Mathematics, 93:173-177, 1989.

16. Kayal, N., Saxena, N.: Complexity of ring morphism problems. Computational Complexity, 15:342-390, 2006. doi:10.1007/s00037-007-0219-8

17. Laison, J.D., Qing, Y.: Subspace intersection graphs. Discrete Mathematics, 310:3413-3416, 2010. doi:10.1016/j.disc.2010.06.042

18. T.A. McKee and F.R. McMorris. Topics in Intersection Graph Theory. SIAM Monographs on Discrete Mathematics and Applications, 1999. doi:10.1137/1. 9780898719802

19. Ngoc, N.K., Bien, M.H., Hai, B.X.: Free subgroups in almost subnormal sub-groups of general skew linear groups. Algebra i Analiz, 28:220-235, 2016. translation in St. Petersburg Mathematical Journal, 28(5):707-717, 2017. doi:10.1090/spmj/1468

20. Pawlik, A., Kozik, J., Krawczyk, T., Lasoń, M., Micek, P., Trotter, W.T., Walczak, B.: Triangle-free intersection graphs of line segments with large chromatic num- ber. Journal of Combinatorial Theory, Series B, 105:6-10, 2014. doi:10.1016/j.jctb.2013. 11.001

21. Penrose, M.: Random Geometric Graphs. Oxford University Press, 2003. doi:10. 1093/acprof:oso/9780198506263.001.0001

22. Yaraneri, E.: Intersection graph of a module. Journal of Algebra and Its Applications, 12:1250218, 2013. doi:10.1142/S0219498812502180 